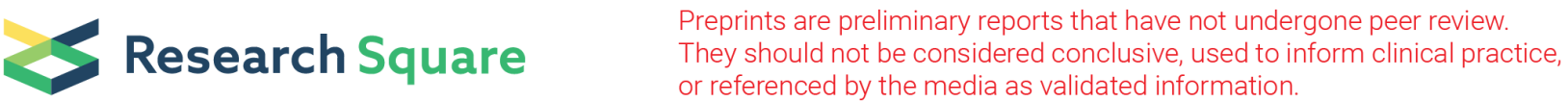 \\ The Effect of Acetate Ringer's Solution Versus lactate Ringer's Solution on Acid Base Physiology in Infants with Biliary Atresia
}

\section{Xiang Liu}

Hebei medical university affiliated children's hospital of hebei province

Hongyan Cao

Hebei medical university affiliated children's hospital of hebei province

Xiaona Tan

Hebei medical university affiliated children's hospital of hebei province

Jing Shi

Hebei medical university affiliated children's hospital of hebei province

\section{Li Qiao}

Hebei medical university affiliated children's hospital of hebei province

\section{Qi Zhang}

Hebei medical university affiliated children's hospital of hebei province

\section{Lei Shi ( $\nabla$ shileilx@163.com )}

Hebei medical university affiliated children's hospital of hebei province

\section{Research Article}

Keywords: Sodium Acetate, acid base balance, lactate, children

Posted Date: August 11th, 2021

DOl: https://doi.org/10.21203/rs.3.rs-745650/v1

License: (c) (i) This work is licensed under a Creative Commons Attribution 4.0 International License.

Read Full License

Version of Record: A version of this preprint was published at BMC Pediatrics on December 1st, 2021. See the published version at https://doi.org/10.1186/s12887-021-03074-4. 


\section{Abstract}

Backgroud: The choice of the perioperative crystalloid is a key component of the fluid management and must take into account the liver function and the appearing metabolic disorders to avoid increase the liver extra metabolism. The aim of this study is to analyze the effect of acetate Ringer's solution or lactate Ringer's solution in biliary atresia patients.

Methods: We included 68 infant patients aged between $21 \sim 65 \mathrm{~d}$, ASA physical status II or $\otimes$, who underwent elective Kasai hepatoportoenterostomy, received either AR and LR for intravenous fluid resuscitation according to their group allocation. Lactate concentration, serum electrolytes and $\mathrm{pH}$ were noteded before skin incision $\left(T_{1}\right)$, end of surgery $\left(T_{2}\right)$ and postoperative $12 \mathrm{~h}$. We also recorded the time of operation, stay of hospital, loss of blood and urinary, total volume of infusion of crystalloid.

Results: Lactate level was significantly higher in Group LR than in Group AR patients at $T_{2}(0.76 \pm 0.13$ versus $0.57 \pm 0.22, P=0.03)$. Compared with $T_{3}$, sodium and chlorine were significantly higher in two groups at $T_{2}(145.2 \pm 3.1$ versus $143.4 \pm 3.4$ and $104.6 \pm 3.7$ versus $105.2 \pm 2.1)$. No significant differences were noted in potassium, $\mathrm{HCO}_{3}{ }^{-}$and calcium. There was no statistically significant difference in $\mathrm{pH}$. No glycopenia was recorded in two groups. No significant difference was noted in administration of vasoactive drug $(0.7 \%$ versus $1 \%)$.

Conclusions: Resuscitation with AR and LR is associated with similar clinical improvement in infants with biliary atresia. Use of AR reduced the level of lactate in comparison with LR.

\section{Introduce}

The aim of perioperative fluid therapy is to provide adequate intravascular volume to ensure tissue perfusion and cellular oxygenation that is the physiologic goal independent of the type of surgery. Biliary atresia is a neonatal liver disease characterized by progressive obstruction and fibrosis of the extrahepatic biliary tree as well as fibrosis and inflammation of the liver parenchyma ${ }^{[1]}$. The current treatment for biliary atresia involves sequential surgical intervention with the Kasai hepatoportoenterostomy ${ }^{[2]}$. The great challenge is that multiple factors affect the perioperative fluid management that may change the acid base balance and electrolyte in Biliary atresia. The choice of the perioperative crystalloid is a key component of the fluid management and must take into account the liver function and the appearing metabolic disorders to avoid increase the liver extra metabolism. The World Health Organization advocates lactate Ringer's solution ( LR ) as the preferred fluid for correction of severe diarrheal dehydration ${ }^{[3]}$. The compositional properties have continued to sustain lactate Ringer's solution as the seemingly ideal resuscitation fluid. Recent critical review has nevertheless brought lactate Ringer's use into question, such as hepatic mediated metabolism, and increased aerobic demand ${ }^{[4]}$.

Acetated Ringer's solution ( AR ) do not display these shortcomings. Acetate as an alternative anion has been proposed ${ }^{[5]}$. Its advantages over standard lactate include its aqueous solubility, stability at high 
concentrations, inert bioactivity and smaller molecular weight. Unlike lactate, acetate is also more rapidly metabolised with less oxygen demand and extra hepatic ${ }^{[6]}$. To date, and to our knowledge, there is a paucity of evidence in the literature on comparative influence of acetate Ringer's solution or lactate Ringer's solution in biliary atresia patients. The primary main objectives of this randomized controlled trial was to compare the effect of acetate Ringer's solution or lactate Ringer's solution on lactate and $\mathrm{HCO}_{3}{ }^{-}$level in biliary atresia patients. The secondary objectives included determination of arterial $\mathrm{pH}$, electrolytes, body temperature, and urine volume, use of vasoactive drugs and hypoglycemia.

\section{Methods}

\section{Study Design}

This randomized, double-blind controlled trial was approved by the Hospital Ethics Committee ( Hebei Medical University affiliated Children's Hospital of Hebei Province, 2019103 ) and was registered with ClinicalTrials ( ChiCTR2000041129). Written informed consent was obtained from the parents of all patients during the preoperative visit. We included 68 infant patients aged between $21 \sim 65 d$, ASA

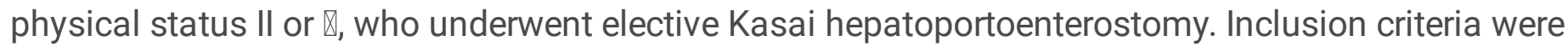
contained to receive treatments, no respiratory distress syndrome and renal failure. Exclusion criteria were fever, abnormal coagulation function, anemia, complicated congenital heart disease requiring emergency cardiac surgery. We had a preoperative liver protection therapy and maximally decreased the alanine transaminase and aspartate aminotransferase concentrations. We randomly assigned eligible patients using computer generated variable block randomization, concealed by sequentially assigned sealed randomization envelopes, to receive either $L R$ and AR. Sequence generation and preparation of randomization envelopes were completed by Q.Z. independent of the research team and who had no further role in the trial. Envelopes were opened immediately before induction of anesthesia by L.Q. who was aware of the randomization outcome only after the envelope was opened.

\section{Anesthesia Management}

The patient was placed on a thermostatically controlled warming blanket and monitoring attached ( electrocardiography, noninvasive blood pressure, pulse oximetry and $\mathrm{P}_{\mathrm{ET}} \mathrm{CO}_{2}$ ) prior to induction. Invasive blood pressure and central venous pressure were implemented after endotracheal intubation. Hemodynamics was monitored using a device (Mostcare care, Italy)via arterial catheterization. The core temperature was monitored continuously with the probe which was set on the pharynx nasalis. General anesthesia was induced by intravenous administration of propofol 1 to $2 \mathrm{mg} / \mathrm{kg}$ and fentanyl $2 \sim 3 \mu \mathrm{g} /$ $\mathrm{kg}$. Neuromuscular blockade was produced by cisatracurium. Patients were intubated with a plain endotracheal tube ( appropriate size 3.5 or 4.0 ). Anesthesia was maintained with inhalation of $0.5 \%$ 1\% sevoflurane in oxygen and air $\left(\mathrm{FiO}_{2} 45 \%\right)$ and remifentanil $0.25 \sim 0.3 \mu \mathrm{g} / \mathrm{kg} / \mathrm{min}$. Controlled mandatory ventilation (Dräeger Fabius, Germany ) with oxygen-air mixture was used with a programmed inspiratory tidal volume of 8 to $10 \mathrm{ml} / \mathrm{kg}$. $\mathrm{P}_{\mathrm{ET}} \mathrm{CO}_{2}$ was maintained between 35 and $45 \mathrm{mmHg}$ by adjusting the 
respiratory rate. The ventilation was delivered with a fixed inspiratory to expiratory ratio of 1:1.5. Respiratory parameters were adjusted or medicated according to the results of blood gas analysis.

Fluid therapy

Patients received either LR or AR for intravenous fluid resuscitation according to their group allocation. Holliday and Segar protocol is still widely used as the most common formula to calculate fluid volume in the intravenous period. According to this calculation, the amount of fluid to be given is $4 \mathrm{ml} / \mathrm{kg}$ for the first 10 kilograms of the child, $2 \mathrm{ml} / \mathrm{kg}$ in addition to $40 \mathrm{ml}$ and $1 \mathrm{ml} / \mathrm{kg}$ in addition to $60 \mathrm{ml}$, calculated with the formula $4 / 2 / 1$. The 3 rd space loss is roughly estimated as $2 \mathrm{ml} / \mathrm{kg} / \mathrm{h}$ for superficial surgery, 4 $7 \mathrm{ml} / \mathrm{kg} / \mathrm{h}$ for thoracotomy and $5 \sim 10 \mathrm{ml} / \mathrm{kg} / \mathrm{h}$ for abdominal surgery ${ }^{[7]}$. Arterial blood gas was then collected every $1 \mathrm{~h}$ until the end of surgery. Trigger for blood transfusion depends on age, hemoglobin level and associated disease states. Based on the patient blood management programs restrictive hemoglobin thresholds may be indicated in infants and children ( target of $7 \mathrm{~g} / \mathrm{dL}$ for haemodynamically stable patient ). The hemoglobin was measured and blood transfusion was determined in time to ensure adequate oxygen supply. The choice of specific agents and interventions was implemented to the discretion of the attending physician specialists.

\section{Data Collection}

Blood gas analysis was performed before skin incision ( $T_{1}$ ), end of surgery $\left(T_{2}\right)$ and postoperative $12 \mathrm{~h}$ $\left(T_{3}\right)$. A standard blood gas analyzer (ABL 800 FLEX, Denmark) was used. The blood glucose concentration was detected and the incidence of perioperative hypoglycemia ( blood glucose $₫ 2.8 \mathrm{mmol} / \mathrm{L}$ ) was recorded. The glucose solution was injected reasonably to treat hypoglycemia. Lactate concentration, serum electrolytes, and $\mathrm{pH}$ of the patients were documented from blood gas. During the anesthesia, we also recorded the time of operation, stay of hospital, loss of blood and urinary, red blood cell transfusions and total volume of infusion of crystalloid. Intraoperative body temperature was monitored, and intraoperative vasoactive agents and hypoglycemia were recorded.

\section{Statistical analysis}

All data were recorded using a standardized data collection sheet and analyzed using the statistical software SPSS Statistics 18 ( SPSS Inc, Chicago, IL, USA ). On the basis of the

results of a previous study, we assumed that the difference between the groups with respect to the primary outcome of lacetate concentration would be $0.12 \mathrm{mmol} / \mathrm{L}$ with a standard deviation of sample difference 0.36 , and thus, 32 patients were required per sequence to achieve the desired power of $90 \%$ ( $\beta$ $=0.10)$ at the $5 \%(a=0.05)$ level of significance. Based on this configuration, the study was designed to enroll 68 patients. The Shapiro-Wilk test was used to test for normality. Because most of the data were normally distributed, they are presented as mean \pm standard deviation. Continuous data, if normally distributed, were compared by Student $t$ test and, if abnormally distributed, were compared by Friedman test to describe changes of measurement parameters within a group during the course of time (three points of measurement ). In order to compare differences between the groups an ANOVA was followed by 
a Student $t$ test. Nonparametric variables were compared between the groups using $\chi 2$ test (Fisher exact test if cell frequencies were small ). All tests were 2 -tailed, and a $P$ value $<0.05$ was considered as significant.

\section{Results}

During the study period, 68 children were assessed, and 60

were randomized ( Figure 1 ). The two groups had comparable demographic characters such as age, weight, sex, time of operation, stay of hospital, loss of blood, red blood cell transfusions, urinary and total volume of infusion of crystalloid. We did not notice significant differences concerning patients' demographic data ( Table 1 ). There were also no significant differences between the groups in coagulation function ( PT, Prothrombin time ; APTT, Activated partial thromboplastin time ) and hepatic function ( ALT, Alanine transaminase; AST, Aspartate aminotransferase ) ( Table 2 ).

Lactate and serum electrolytes levels were compared between the two groups at different time points. Lactate level was significantly higher in Group LR than in Group AR patients at $T_{2}(0.76 \pm 0.13$ versus $0.57 \pm 0.22, P=0.03$ ) ( Table 4 ). Compared with $\mathrm{T}_{3}$, sodium and chlorine were significantly higher in two groups at $\mathrm{T}_{2}$ ( $145.2 \pm 3.1$ versus $143.4 \pm 3.4$ and $104.6 \pm 3.7$ versus $105.2 \pm 2.1$ ) ( Table 3 ). No significant differences were noted in potassium, $\mathrm{HCO}_{3}{ }^{-}$and calcium. Temperature was significantly higher at $\mathrm{T}_{3}$ in two groups (37.2 \pm 0.31 and $37.3 \pm 0.37$ ). There was no statistically significant difference in $\mathrm{pH}$ ( Table 4 ).

No glycopenia was recorded in the two groups. No significant difference was noted in administration of vasoactive drug ( $0.7 \%$ versus $1 \%$ ) ( Table 1 ).

\section{Discussion}

The main findings of this study are the demonstration that acetate Ringer's solution is safe as a resuscitation medium, and further, that it might have some clinical advantages when compared to lactate Ringer's solution as a control group.

Administration of intravenous solutions required to correct physiological functions that have been altered due to surgical stress and anesthetic agents and to maintain body homeostasis to provide oxygen to the tissues $^{[8]}$. In this way, the fluid deficiency is replaced, sufficient tissue perfusion is provided and the unwanted effects of anesthetics are tried to be removed.

High lactate level have been associated with poor outcomes in the critically ill patients ${ }^{[9]}$. Lactate was initially introduced as an alkali. Its alkalinizing effect depends on its reutilization for glucose synthesis and its oxidative degradation to $\mathrm{H}_{2} \mathrm{O}$ and $\mathrm{CO}_{2}$, which converts into bicarbonate. Abnormalities of lactate 
metabolism are very common in patients undergoing prolonged surgery. The metabolism of lactate is dependent on the kidney and liver, and as such, when the functions of these organs are compromised, there will be lactate accumulation ${ }^{[10]}$. Although we observed that lactate level was significantly higher in Group LR than in Group AR at the end of surgery, it was within the normal range. We hypothesised that liver function was limited to metabolise the lactate with biliary atresia patients and the lactate was accumulated, but the liver has a functional reserve and can metabolise lactate. Acetate is metabolized more efficiently in peripheral tissues. Its use in intravenous solutions is becoming popular, because it readily convertible to bicarbonate than lactate. The main organ that metabolizes lactate is liver, whereas acetate can be metabolized widely throughout the body and is not mainly dependent on the liver. In addition, acetate can be metabolized more quickly than lactate. This study showed no difference between the two groups in bicarbonate. In contrast, Kumar et al used acetated crystalloid as an intraoperative fluid and the levels of bicarbonate and base excess showed an improved profile ${ }^{[11]}$. Increase in lactate level is commonly observed if the volume of liver is inadequate following major hepatectomy ${ }^{[12]}$. Sunil et al found that the level of lactate in the lactated Ringer's group was significantly higher than in the acetate solution group at the end of the operation ${ }^{[13]}$. In line with our result , acetated Ringer's solution was found to be safer as compared to normal saline in protecting young children undergoing major surgery against the risk of increasing plasma chlorides and the subsequent metabolic acidosis ${ }^{[14]}$.

This study showed that although serum chloride and serum sodium were significant higher at the end of surgery, the levels were within the normal range in both groups. The contribution of hyperchloremia toward persistent acidosis, however, did not seem to play a major role in our study population. Khan et al found that lactate Ringer's solution to prevent hyperchloremic metabolic acidosis ${ }^{[15]}$. We demonstrated that AR and LR may play a less role in electrolyte disorders including hyperchloremic and hypernatremia with fluid resuscitation. Concerns about intravenous hypotonic fluids have focused on potential neurological sequelae associated with severe hospital-induced hyponatremia ${ }^{[16]}$. Hyponatremia is the most common electrolyte disorder in children, affecting approximately $25 \%$ of hospitalized children and $30 \%$ of children in the postoperative period, most of which occurred after uncomplicated surgeries ${ }^{[17]}$. Hyponatremic encephalopathy is the most crucial risk of acute hyponatremia and may result in permanent neurological damage or death. At least half of the cases documented with the diagnosis of hyponatremia in children occurred in the postoperative period, most of which occurred after uncomplicated surgeries. Stimulation of the antidiuretic hormone $(A D H)$ may be due to hemodynamic causes such as hypovolemia and hypotension, other factors that cause hemodynamic-independent nonosmotic $A D H$ release include postoperative status, positive pressure ventilation, pain, nausea, vomiting and the use of narcotic medication ${ }^{[18]}$. As a result of decreased diuresis effect of kidney due to ADH over stimulation, fluid retention and related dilutional hyponatremia increase the risk of hyponatremic encephalopathy in pediatric patients in perioperative period ${ }^{[19]}$.

The hyponatremia was not found in both groups. Hence, we can draw the conclusion that administration of acetate Ringer's solution or lactate Ringer's solution may play a less role in leading hyponatremia in infants with biliary atresia. Intraoperative hypothermia is associated with numerous 
complications such as decreased drug metabolism, impairment of coagulation, and shivering ${ }^{[20]}$. In our study, we observed that the temperature was a signifcantly higher at postoperative $12 \mathrm{~h}$ in two groups. The temperature may be related to postoperative fever ${ }^{[21]}$. The inflammatory mechanisms accountable for postoperative fever have been the subject of a number of studies. Tissue damages alone results in the disruption of phospholipids from the cell membrane, leading to a cascade of prostaglandins and cytokines which ultimately lead to a body temperature elevation. There was no significant differences between the groups at other time points. We used forced-air prewarming before anesthesia induction to prevent the development of hypothermia. Hypothermia was not observed in infant patients.

The incidence of hypoglycemia during induction of anesthesia is reported to be between $0 \%$ and $2.5 \%$. In most of the children identified with hypoglycemia, an average of 10 hours of fasting times are reported. Hypoglycemia was not observed in children who had drunk clear fluid up to 2 hours before surgery. ${ }^{[22]}$. Our study found that no hypoglycemia was recorded following fluid resuscitation in both groups. All infants patients received clear fluids containing $3 \mathrm{ml} / \mathrm{kg}$ glucose over the last 2 hour before surgery, according to their weight calculated in our study ${ }^{[23]}$.

Limitations of the study include small sample size, lack of comparison of base excess. The data should have been collected up to postoperation $24 \mathrm{~h}$. Postoperation coagulation function and hepatic function have not been assessed and analyzed. Hemodynamics have not been analyzed during operation. Only a single surgery was included in the study. Further research involving larger number of patients undergoing different surgeries is needed to know the acid base physiology in infant or neonatel patients.

In summary, resuscitation with AR and LR is associated with similar clinical improvement in infant with biliary atresia. Use of acetate Ringer's solution reduced levels of lactate in comparison with LR. Hence, AR is to be considered as the fluid of choice owing to the clinical improvement with the Kasai hepatoportoenterostomy.

\section{Abbreviation}

AR囚acetate Ringer's solution

LR囚lactate Ringer's solution

$\mathrm{P}_{\mathrm{ET}} \mathrm{CO}_{2}$ \end-tidal carbon dioxide partial pressure

PT囚prothrombin time

APTT囚activated partial thromboplastin time

ALT囚alanine transaminase

AST囚aspartate aminotransferase 


\section{Declarations}

\section{Ethics approval and consent to participate}

Trial was approved by Hospital Ethics Committee Hebei medical university affiliated children's hospital of hebei province (NO: 2019103) and registered before patient enrollment in the Chinese Clinical Trial Registry (ChiCTR2000041129).

\section{Consent for publication}

We consent to publish

\section{Availability of data and material}

Our raw data can be shared dy a public repository.

https://pan.baidu.com/s/1N3oLoM3Fq9j6Lbf8dM2i8g

\section{Competing interests}

None of the authors report any competing interest

\section{Funding}

None

\section{Authors' contributions}

Xiang Liu was the principal investigator who involved in data collection, data analysis, data interpretation, and manuscript preparation. Hongyan Cao was involved in data collection. Xiaona Tan was involved in data analysis. Li Qiao and Qi Zhang were involved in the study design, data analysis, data interpretation. Jing Shi was involved in the study design. Shi Lei was involved in the study design and coordinated the study and manuscript preparation.

\section{Acknowledgements}

We thank the surgeon Xu and nurse Li who served as scientific advisors and provided care for study patients. 


\section{References}

1. Schreiber RA. Newborn Screening for Biliary Atresia. JAMA. 2020; 323(12):1137-1138.

2. Shneider BL, Magee JC, Karpen SJ, et al. Total Serum Bilirubin within 3 Months of Hepatoportoenterostomy Predicts Short-Term Outcomes in Biliary Atresia. J Pediatr. 2016; 170: 211217.e1-2.

3. World Health Organization. The treatment of diarrhea: a manual for physicians and other senior health workers. Geneva, Switzerland: WHO, 2005. Available at:

http://www.who.int/maternal_child_adolescent/documents/9241593180/en/. Accessed on August 15, 2014.

4. Guidet B, Soni N, Della RG, et al. A balanced view of balanced solutions. Crit Care. 2010; 14:325.

5. Rajan S, Srikumar S, Tosh P, et al. Effect of lactate versus acetate-based intravenous fluids on acidbase balance in patients undergoing free flap reconstructive surgeries. J Anaesthesiol Clin Pharmacol. 2017; 33:514-9.

6. Ellekjaer KL, Perner A, Sivapalan P, et al. Acetateâ vs lactate buffered crystalloid solutions: Protocol for a systematic review with metaâ analysis and trial sequential analysis. Acta Anaesthesiol Scand. 2020; 65(1):123-127.

7. APA Consensus Guideline On Perioperative Fluid Management In Children. V 1.1 September 2007 Apagbi Review Date August 2010.

8. Tomi Myrberg, Linnea Lindelöf, Magnus Hultin. Effect of preoperative fluid therapy on hemodynamic stability during anesthesia induction, a randomized study. Acta Anaesthesiol Scand. 2019; 63(9):1129-1136.

9. Nazer LH, Rimawi D, Hawari FI. Evaluating the Predictive Value of Lactate in Patients With Cancer Having Septic Shock. J intensive care med. 2020; 35(8):789-796.

10. Gladden LB. Lactate metabolism: A new paradigm for the third millennium. J Physiol. 2004;558:530.

11. Kumar L, Seetharaman M, Rajmohan N, et al. Metabolic profle in right lobe living donor hepatectomy: Comparison of lactated Ringer's solution and normal saline versus acetate based balanced salt solution a pilot study. Indian J Anaesth. 2016; 60(10): 719-725.

12. Marko P, Gabrielli A, Caruso LJ. Too much lactate or too little liver? J Clin Anesth. 2004;16:38995.

13. Rajan S, Srikumar S, Tosh P, et al. Effect of lactate versus acetate-based intravenous fluids on acidbase balance in patients undergoing free flap reconstructive surgeries. J Anaesthesiol Clin Pharmacol. 2017;33:514-9.

14. Disma N, Mameli L, Pistorio A, et al. A novel balanced isotonic sodium solution vs. normal saline during major surgery in children up to 36 months: A multicenter RCT. Paediatr Anaesth. 2014;24:9806.

15. Khan MF, Siddiqui KM, Asghar MA. Fluid choice during perioperative care in children: A survey of present-day proposing practice by anesthesiologists in a tertiary care hospital. Saudi J Anaesth. 2018; 12(1):42-45. 
16. Eulmesekian PG, Perez A, Minces PG, et al. Hospital-acquired hyponatremia in postoperative pediatric patients: Prospective observational study. Pediatr Crit Care Med. 2010; 11(4): 479-483.

17. Holliday MA, Friedman AL, Segar WE, et al. Acute hospital-induced hyponatremia in children: a physiologic approach. J Pediatr. 2004; 145(5):584-587.

18. Park KS, Yoo KY. Role of vasopressin in current anesthetic practice. Korean J Anesthesiol. 2017; 70(3):245-257.

19. Khan MF, Siddiqui KM, Asghar MA. Fluid choice during perioperative care in children: A survey of present-day proposing practice by anesthesiologists in a tertiary care hospital. Saudi J Anaesth. 2018; 12(1):42-45.

20. Sarti A, Recanati D, Furlan S. Thermal regulation and intraoperative hypothermia. Minerva anestesiol. $2005 ; 71: 379-383$.

21. Maday KR, Hurt JB, Harrelson P, et al. Evaluating postoperative fever. JAAPA. 2016; 29 (10):23-28.

22. Frykholm $P$, Schindler E, Sumpelmann R. Pre-operative fasting in children. A review of the existing guidelines and recent developments. Br J Anaesth. 2018; 120(3):469-474.

23. Schmidt A, Buehler P, Seglias $L$, et al. Gastric $\mathrm{pH}$ and residual volume after 1 and $2 \mathrm{~h}$ fasting time for clear fluids in children. Br J Anaesth. 2015; 114(3):477-482.

\section{Tables}

Table 1 atient characteristics and the comparative data between the two groups

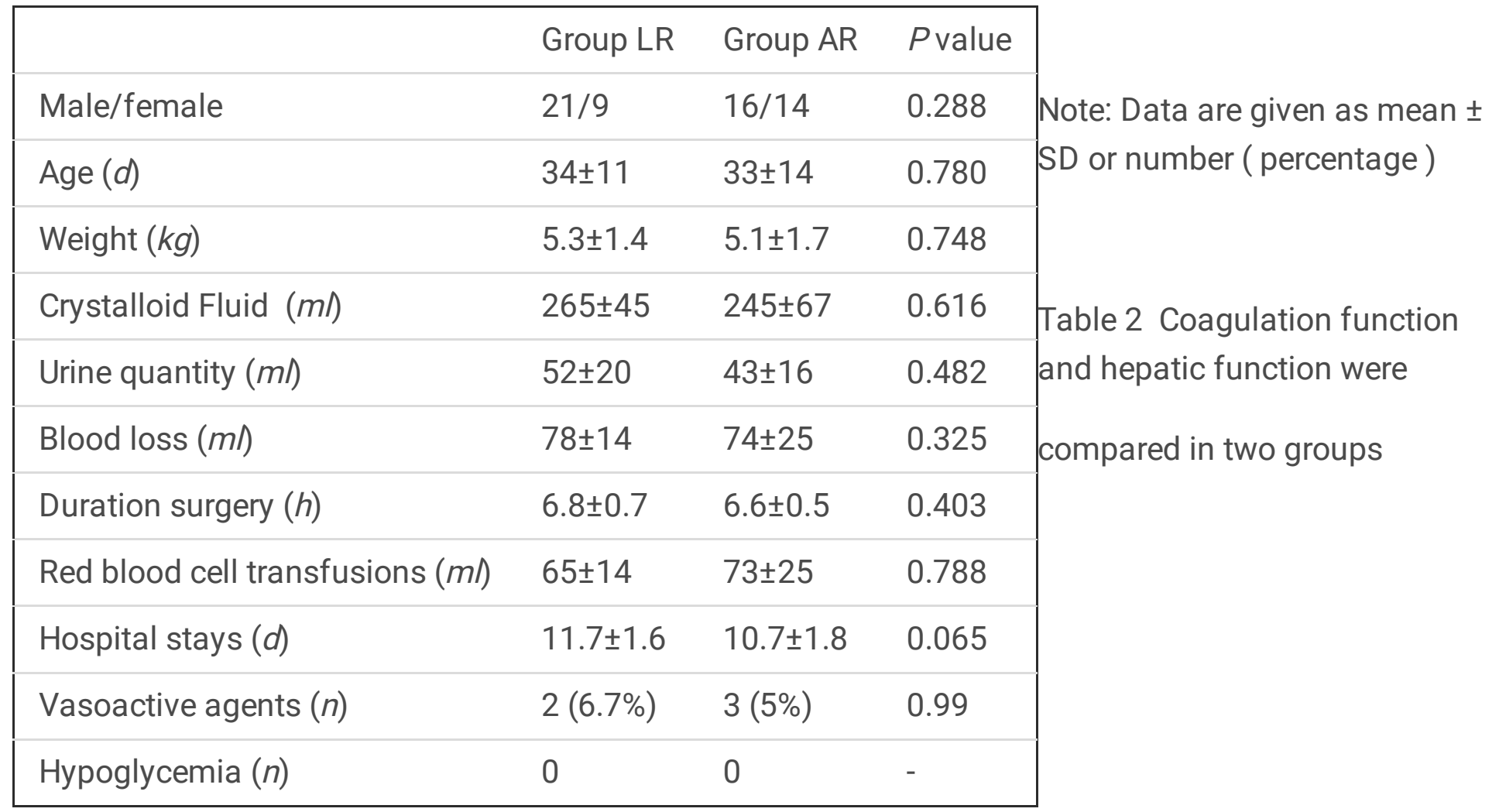




\begin{tabular}{|llll|}
\hline & Group LR & Group AR & $P$ value \\
\hline ALT $(U / L)$ & $102 \pm 12$ & $97 \pm 25$ & 0.533 \\
\hline AST $(U / L)$ & $86 \pm 38$ & $75 \pm 24$ & 0.472 \\
\hline APTT $(S)$ & $32.1 \pm 4.5$ & $31.1 \pm 4.7$ & 0.393 \\
\hline PT $(S)$ & $11.3 \pm 0.8$ & $10.1 \pm 0.7$ & 0.375 \\
\hline
\end{tabular}

Note: Data are given as mean \pm SD

ALT: alanine transaminase, AST: aspartate aminotransferase, APTT: activated partial thromboplastin time, PT: prothrombin time

Table 3 Electrolytes levels were compared between the two groups at different time points 


\begin{tabular}{|c|c|c|c|}
\hline \multicolumn{4}{|c|}{$\mathrm{Na}^{+}(\mathrm{mmol} / \mathrm{L})$} \\
\hline Time & LR & AR & $P$ value \\
\hline $\mathrm{T}_{1}$ & $140.3 \pm 2.6$ & $142.5 \pm 2.7$ & 0.129 \\
\hline $\mathrm{T}_{2}$ & $145.2 \pm 3.1^{a}$ & $143.4 \pm 3.4^{\mathrm{a}}$ & 0.203 \\
\hline $\mathrm{T}_{3}$ & $139.2 \pm 4.9$ & $138.5 \pm 2.3$ & 0.734 \\
\hline \multicolumn{4}{|c|}{$\mathrm{K}^{+}(\mathrm{mmol} / \mathrm{L})$} \\
\hline $\mathrm{T}_{1}$ & $4.01 \pm 0.43$ & $3.89 \pm 0.47$ & 0.587 \\
\hline $\mathrm{T}_{2}$ & $4.06 \pm 0.23$ & $3.81 \pm 0.41$ & 0.111 \\
\hline $\mathrm{T}_{3}$ & $3.75 \pm 0.32$ & $4.03 \pm 0.27$ & 0.055 \\
\hline & \multicolumn{3}{|c|}{$\mathrm{Ca}^{2+}(\mathrm{mmol} / \mathrm{L})$} \\
\hline $\mathrm{T}_{1}$ & $1.18 \pm 0.07$ & $1.24 \pm 0.07$ & 0.136 \\
\hline $\mathrm{T}_{2}$ & $1.20 \pm 0.03$ & $1.22 \pm 0.06$ & 0.495 \\
\hline \multirow[t]{2}{*}{$\mathrm{T}_{3}$} & $1.17 \pm 0.06$ & $1.19 \pm 0.05$ & 0.507 \\
\hline & \multicolumn{3}{|c|}{$\mathrm{Cl}^{-}(\mathrm{mmol} / \mathrm{L})$} \\
\hline $\mathrm{T}_{1}$ & $102.4 \pm 3.7$ & $104.1 \pm 2.1$ & 0.212 \\
\hline $\mathrm{T}_{2}$ & $104.6 \pm 3.2^{b}$ & $106.4 \pm 2.0^{b}$ & 0.753 \\
\hline $\mathrm{T}_{3}$ & $100.7 \pm 1.8$ & $101.4 \pm 2.9$ & 0.617 \\
\hline
\end{tabular}

Note: Data are given as mean $\pm \mathrm{SD}$

a,b $P<0.05$ versus $\mathrm{T}_{1}$ and $\mathrm{T}_{3}$ in $\mathrm{Na}^{+}$and $\mathrm{Cl}^{-}$

Table 4 Temperature, $\mathrm{pH}, \mathrm{HCO}^{-}$and lactate levels were compared between the two groups at different time points 


\begin{tabular}{|c|c|c|c|}
\hline \multicolumn{4}{|c|}{ Temperature $\left({ }^{\circ} \mathrm{C}\right)$} \\
\hline Time & LR & AR & $P$ value \\
\hline $\mathrm{T}_{1}$ & $36.8 \pm 0.23$ & $36.8 \pm 0.25$ & 0.625 \\
\hline $\mathrm{T}_{2}$ & $36.8 \pm 0.17$ & $36.9 \pm 0.27$ & 0.093 \\
\hline $\mathrm{T}_{3}$ & $37.2 \pm 0.31^{*}$ & $37.3 \pm 0.37 *$ & 0.746 \\
\hline \multicolumn{4}{|c|}{$\mathrm{pH}$} \\
\hline $\mathrm{T}_{1}$ & $7.37 \pm 0.05$ & $7.38 \pm 0.05$ & 0.790 \\
\hline $\mathrm{T}_{2}$ & $7.40 \pm 0.04$ & $7.37 \pm 0.06$ & 0.207 \\
\hline $\mathrm{T}_{3}$ & $7.40 \pm 0.05$ & $7.39 \pm 0.04$ & 0.476 \\
\hline \multicolumn{4}{|c|}{$\mathrm{HCO}_{3}{ }^{-}(\mathrm{mmol} / \mathrm{L})$} \\
\hline $\mathrm{T}_{1}$ & $22.4 \pm 2.0$ & $21.5 \pm 2.4$ & 0.707 \\
\hline $\mathrm{T}_{2}$ & $20.1 \pm 1.8$ & $20.9 \pm 3.1$ & 0.796 \\
\hline $\mathrm{T}_{3}$ & $22.1 \pm 1.6$ & $21.9 \pm 1.1$ & 0.743 \\
\hline \multicolumn{4}{|c|}{ Lactate $(\mathrm{mmol} / \mathrm{L})$} \\
\hline $\mathrm{T}_{1}$ & $1.09 \pm 0.34$ & $0.92 \pm 0.38$ & 0.343 \\
\hline $\mathrm{T}_{2}$ & $0.76 \pm 0.13^{\#}$ & $0.57 \pm 0.22$ & 0.030 \\
\hline $\mathrm{T}_{3}$ & $1.05 \pm 0.25$ & $0.92 \pm 0.21$ & 0.229 \\
\hline
\end{tabular}

Note: Data are given as mean \pm SD

${ }^{*} P<0.05$ versus $\mathrm{T}_{1}$ and $\mathrm{T}_{2}$ in temperature

${ }^{\#} P<0.05$ versus AR in lactate

Figures 


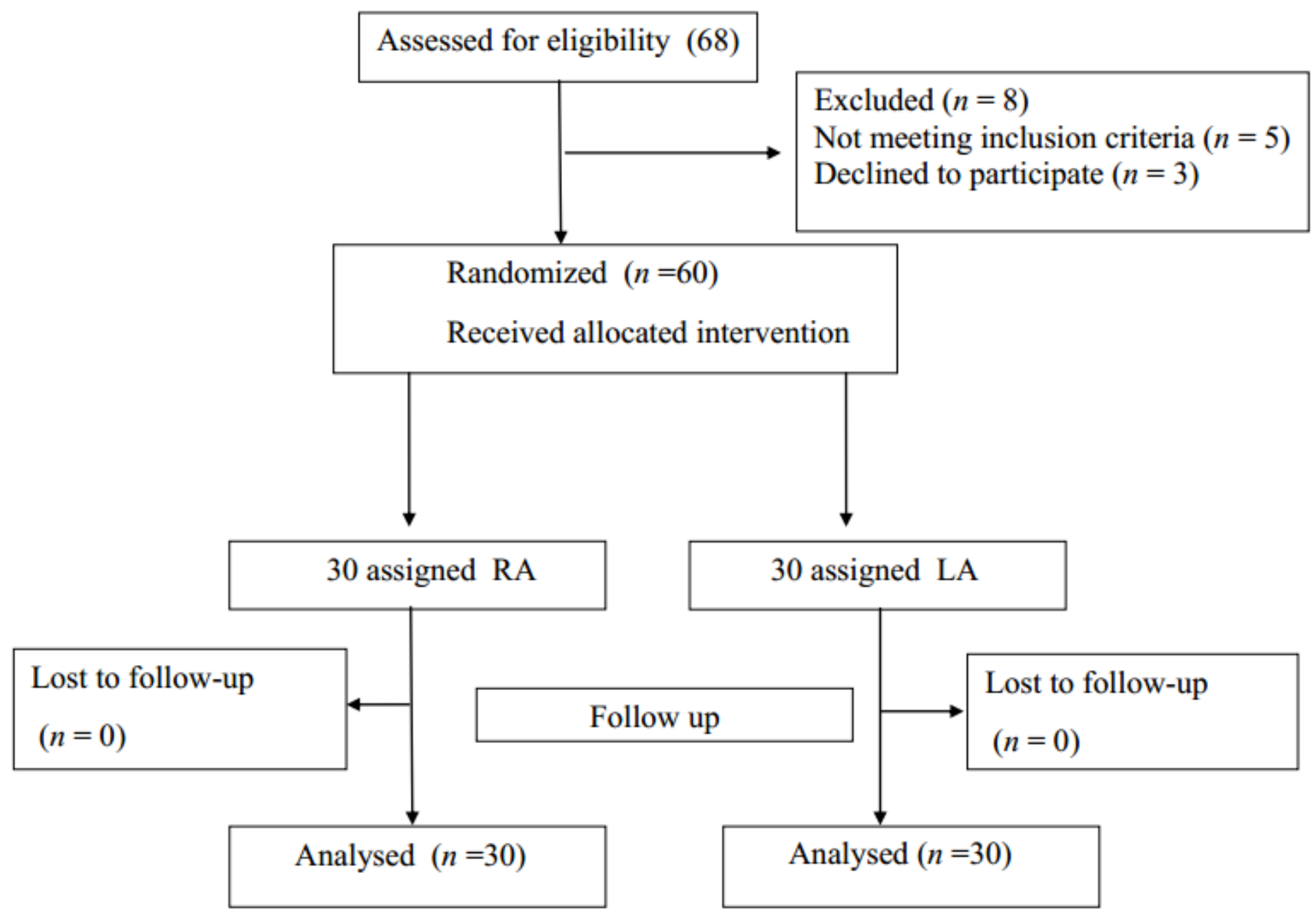

Figure 1

Consort flow chart. A total of 68 children were enrolled and completed the study. 